\title{
Evaluation of X-ray table mattresses for radiation attenuation and impact on image quality
}

\author{
Nadi Alreshedi \\ Dr Lucy Walton \\ Prof Peter Hogg \\ Joanne Webb \\ Dr Andrew Tootell
}

\section{Abstract}

Introduction

Mattresses in the radiology department tend to be an overlooked aspect of imaging equipment. This paper evaluates the radiation attenuation characteristics of mattresses and the effect they have on image quality.

Method

Thirteen mattresses (from new to 20 years of age) were evaluated. Incident air kerma (IAK) was measured in two conditions, with and without mattress over a range of exposure factors using a digital dosimeter. The percentage change was calculated and applied to the set mAs to illustrate the "effective mAs" delivered to an image receptor. Image quality was assessed by calculating the inverse image quality factor (IQF $F_{\text {inv }}$ ) using a commercially available phantom (CDRAD) for the same exposure factors. The correlation of age and attenuation and image quality was calculated.

Results

Measured IAK and image quality was affected by the addition of a mattress. IAK decreased due to attenuation and IQFinv indicated worse image quality. IAK correlated negatively with mattress age indicating that older mattresses have higher attenuation properties. The clinical impact for radiation increase was insignificant as it resulted in an average of 0.05 change in mAs. There was no correlation between age and image quality.

Conclusion

The results indicate that while the presence of a mattress does impact on transmitted radiation and the quality of the image, the clinical impact is insignificant. Attenuation correlates with age but with no clinical significance. There is no correlation between age and image quality.

Implications for practice

Quality control tests for attenuation and impact on image quality are not required in clinical practice. The method could be used by manufacturers to test new materials and mattresses and could provide users with specifications of new products. 


\section{Introduction}

Mattresses used in radiology are arguably an overlooked aspect of ancillary imaging equipment. Their role is to facilitate patient comfort and compliance during imaging. In performing these roles, they should attenuate the primary beam minimally and ideally have no effect on the resulting image quality. The work described in this paper compliments research already published from the project that assesses mattresses' abilities to redistribute the pressure of jeopardy areas (head, sacrum and heels) to minimise the development of pressure ulcers in at risk populations during medical imaging (1). The mattresses used in radiology are in the field of view and although they are made from low density materials and are relatively thin, they will attenuate the primary X-ray beam. In turn this could require an increase in $\mathrm{mAs}$ to compensate for absorbed photons, thus increasing patient dose. The materials within the mattresses or their construction should not impact negatively on the diagnostic acceptability of the resulting radiographic image.

Any element of the imaging chain should undergo quality assurance checks and it is argued that mattresses used within radiology should be subjected to checks too (2). Without regular quality assurance of existing mattresses and testing of new products, practitioners do not have evidence to indicate existing equipment requires replacement or manufacturers will not know if their products are fit for purpose. However, there is no published method for testing mattresses' radiation attenuation properties or impact on image quality that can be used during development, at point of purchase or at regular intervals on routinely used mattresses.

This paper describes a method and the results from tests that were carried out on a range of clinically used and new mattresses in relation to their attenuation of the primary beam and objective physical measures of image quality.

\section{Method}

\section{Experimental Design}

The method considered two elements of mattress quality for X-ray imaging, the X-ray attenuation properties over a range of exposure factors, and its effect on image quality. A total of 13 mattresses were evaluated with ages $20,15(x 2), 10(x 5), 8,7(x 2), 6$ and 0 years (new). All mattresses were 25 mm thick

Prior to data collection, the X-ray tube and automatic exposure control (AEC) (Wolverson Acroma, Wolverson X-ray Ltd Willenhall UK) and digital radiography (DR) system (AeroDR, KonicaMinolta, Tokyo, Japan) were tested to ensure performance was within accepted limits. Equipment used to measure incident air kerma and image quality had been calibrated and was operating within tolerances. Quality testing was conducted in accordance with IPEM reports numbers 91 and 32 Part II $(3,4)$.

A range of $k V p$ values from 65 to 110 stepping through $5 \mathrm{kVp}$ increments was used for both image quality and dose data acquisition. The corresponding $m A s$ values were generated using the $A E C$ and a $17.5 \mathrm{~cm}$ thick slab of polymethyl methacrylate (PMMA) representing the thickness of an adult abdomen based on the measurements taken from an anthropomorphic abdomen phantom (PH-5 CT Abdomen Phantom (Kyoto Kagaku, Kyoto, Japan))(Table 1). No mattress was present during data collection. 


\begin{tabular}{|l|l|l|l|l|l|l|l|l|l|l|}
\hline $\mathrm{kVp}$ & 65 & 70 & 75 & 80 & 85 & 90 & 95 & 100 & 105 & 110 \\
\hline $\mathrm{mAs}$ & 40 & 25 & 20 & 14 & 10 & 8 & 6.3 & 5.6 & 4.5 & 4 \\
\hline
\end{tabular}

Table 1.Demonstrates $k V p$ and $m A s$ used to collect dose and image quality data. The $m A s$ was generated by using the AEC at the respective $k V$ to expose a $17.5 \mathrm{~cm}$ thick slab of PMMA representing the thickness of an adult abdomen with no mattress in situ.

\section{Method for Measuring X-ray Attenuation}

The attenuation properties of the $13 \mathrm{x}$-ray mattresses were tested by comparing the incident air kerma IAK in the presence and absence of a mattress. A RaySafe X2 (Unfors RaySafe AB, Billdal, Sweden) with an $\mathrm{R} / \mathrm{F}$ sensor was positioned parallel to the to the table top in the central beam with a source to image distance (SID) of $120 \mathrm{~cm}$ (source to object)/dosimeter distance (SOD) of $100 \mathrm{~cm}$ ) and secured to the table top using tape. Three exposures were made at each exposure factor listed in Table 1 and the mean IAK and standard deviation calculated. A simple subtraction of the IAK with the mattress from the IAK without would provide the dose absorbed by the mattress. The IAK is a measure of the radiation dose the would be transmitted through the mattress. The mattress under test was then placed over the dosimeter and the exposures repeated a further three times and then averaged to minimise random error (Figure 1)

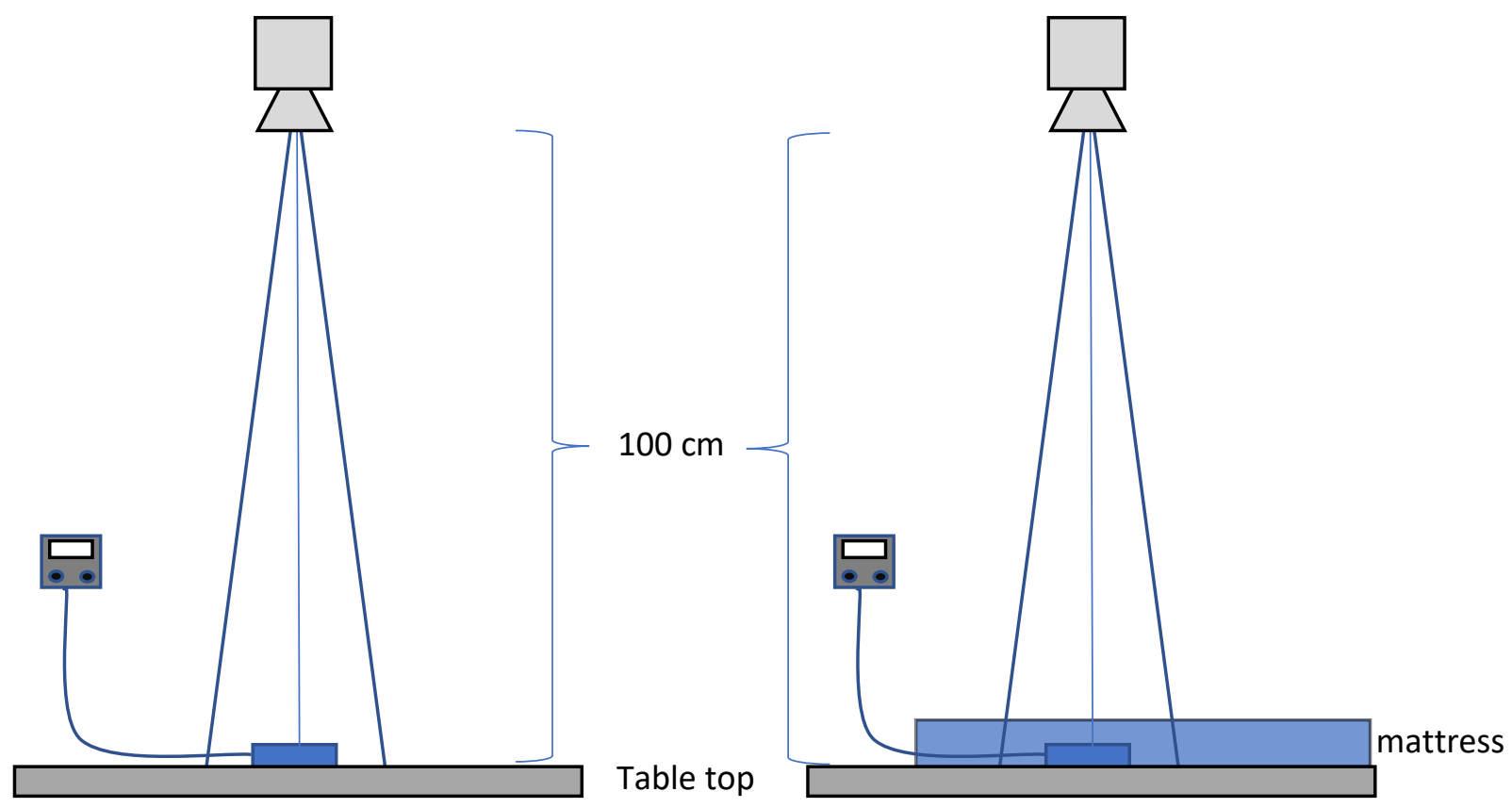

Figure 1 Diagram illustrating the placement of the digital dosimeter with (a) and without (b) mattress.

The correlation of mattress age and IAK was calculated.

\section{Method for Measuring Image quality}

Objective physical image quality measurement was performed using a commercially available contrast detail phantom (CDP), specifically the CDRAD2.0 phantom and analysis software (Artinis Medical Systems, Elnst, Netherlands). Objective measurements of image quality do not suffer from variations that can arise from participants viewing and evaluating image quality and provide reliable and reproducible results that have external validity (5). This reproducibility allows changes over time to be recorded. 
The CDRAD phantom consists of a $10 \mathrm{~mm}$ thick PMMA sheet with a total of 225 holes of varying depths and sizes drilled into it. The diameter of these holes varies from $0.3 \mathrm{~mm}$ up to $8 \mathrm{~mm}$ across 15 depths starting at $0.3 \mathrm{~mm}$ (low contrast) to $8 \mathrm{~mm}$ (high contrast) (Figure 2). The phantom effectively uses the contrast between air and PMMA to create the image contrast $(6,7)$. Image quality is assessed by analysing where the holes are visible with smaller and shallower holes the more difficult to detect.
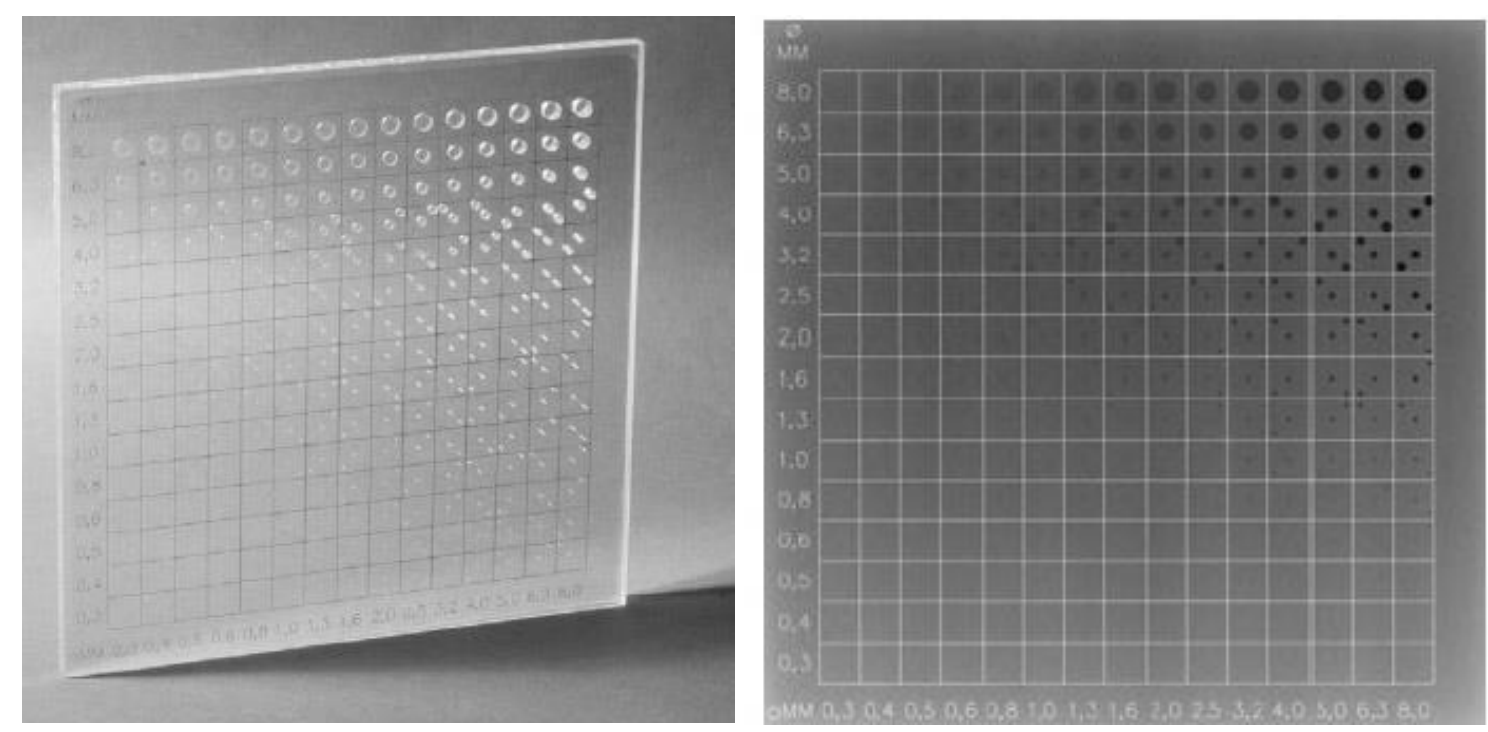

Figure 2 CDRAD phantom and radiographic image (7)

The CDRAD was placed in the centre of the $17.5 \mathrm{~cm}$ block of PMMA to simulate the attenuation and scatter of the abdomen. To simulate clinical practice the DR cassette was placed in the table bucky incorporating an oscillating focussed anti-scatter grid. Images of the CDRAD phantom with and without the mattress were acquired to calculate the change in image quality when the mattress is in the field of view. To allow for random fluctuations in noise, three images for each exposure factor and condition were acquired for analysis and the mean calculated.

The resulting images are exported from the imaging system as DICOM data for import into the CDRAD analyser software. The software generates a contrast detail curve that illustrates the minimum diameter detected for each hole depth (Figure 3). 


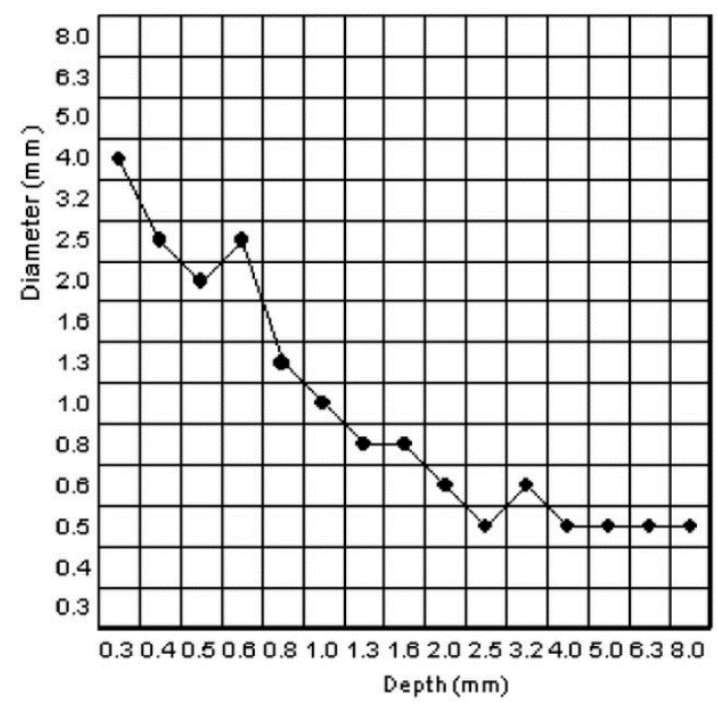

Figure 3 Contrast Detail curve generated by the CDRAD analyser software (5)

Quantification of image quality is performed through the software's calculation of the Inverse Image Quality Factor (IQF $\left.F_{\text {inv }}\right)$. This figure is calculated using the following equation (7):

$I Q F_{\text {inv }}=\frac{100}{\sum_{i=1}^{15} h_{i} \cdot D_{i, \text { threshold }}}$

Where:

- $h_{i}$ refers to the hole depth-column $i$

- $D_{i}$ refers to the minimum diameter (threshold diameter) detected for hole-column column $i$

A detailed description of the analysis is provided in the user guide of the phantom and in Konst et al $(7,8)$. In summary, the image quality figure (IQF) is the sum of the product of the depth of hole and the visible diameter across the 15 columns. A lower IQF represents a higher quality image in that smaller holes and more shallow holes are visible. For the inverse IQF, a higher value indicates higher image quality and represents a figure of merit. With increased image quality, the CD curve will go down (9). Visibility of the holes is determined automatically by the accompanying software (7).

Correlation of mattress age and IQFinv was calculated. 


\section{Results}

Table 2 shows the recorded IAK without the mattress (baseline) and the IAK following attenuation by the mattress. Analysis of percentage decrease is shown in Table 3 and illustrated in Figure 4. The pattern indicates that the older the mattress the greater the attenuation of the X-ray beam. This is supported when the correlation of mattress age and decrease in IAK is compared; there is a moderate/large correlation (0.38-0.51).

\begin{tabular}{|c|c|r|r|r|r|r|r|r|r|r|r|r|r|r|}
\hline Age (years) & Without & $\mathbf{2 0}$ & $\mathbf{1 5}$ & $\mathbf{1 5}$ & $\mathbf{1 0}$ & $\mathbf{1 0}$ & $\mathbf{1 0}$ & $\mathbf{1 0}$ & $\mathbf{1 0}$ & $\mathbf{8}$ & $\mathbf{7}$ & $\mathbf{7}$ & $\mathbf{6}$ & $\mathbf{0}$ \\
\hline 65kV 40mAs & 532.22 & 496.10 & 517.65 & 463.63 & 504.73 & 503.73 & 507.63 & 501.27 & 528.33 & 505.48 & 507.50 & 499.03 & 497.93 & 520.20 \\
70kV 25mAs & 401.97 & 374.93 & 390.97 & 352.57 & 381.58 & 380.82 & 384.20 & 379.43 & 398.00 & 382.87 & 383.82 & 377.57 & 377.33 & 391.28 \\
75kV 20mAs & 382.97 & 357.43 & 371.98 & 337.10 & 363.37 & 362.68 & 365.97 & 361.00 & 378.60 & 365.30 & 365.03 & 359.83 & 362.40 & 372.58 \\
80kV 14mAs & 314.87 & 294.73 & 299.02 & 279.47 & 299.97 & 300.78 & 301.43 & 298.20 & 311.90 & 300.27 & 301.00 & 296.70 & 297.13 & 306.83 \\
85kV 10mAs & 261.85 & 244.57 & 247.57 & 233.03 & 249.00 & 251.22 & 250.87 & 247.80 & 258.50 & 249.52 & 249.58 & 246.37 & 246.50 & 254.05 \\
90kV 8mAs & 237.78 & 222.33 & 226.15 & 212.57 & 226.52 & 227.13 & 228.63 & 225.17 & 234.60 & 227.28 & 233.73 & 224.37 & 224.17 & 230.95 \\
95kV 6.3mAs & 212.27 & 198.73 & 202.77 & 191.23 & 202.73 & 201.97 & 204.47 & 201.60 & 209.60 & 203.10 & 203.13 & 200.47 & 201.10 & 206.30 \\
100kV 5.6mAs & 208.72 & 194.60 & 199.38 & 187.60 & 199.00 & 198.27 & 199.97 & 197.67 & 205.63 & 199.23 & 199.05 & 197.10 & 196.83 & 202.32 \\
105kV 4.5mAs & 187.92 & 173.90 & 180.45 & 163.27 & 179.02 & 177.93 & 179.80 & 176.93 & 184.03 & 179.07 & 179.08 & 177.07 & 177.30 & 181.53 \\
110kV 4mAs & 180.95 & 168.20 & 174.97 & 158.57 & 173.93 & 172.00 & 174.90 & 171.50 & 178.27 & 173.62 & 173.28 & 171.23 & 171.00 & 176.20 \\
\hline
\end{tabular}

Table 2 Recorded IAK without and with mattress present across the range of exposure factors 


\begin{tabular}{|c|c|c|c|c|c|c|c|c|c|c|c|c|c|}
\hline \multicolumn{14}{|c|}{ Percentage decrease in IAK due to attenuation of the mattress (\%) } \\
\hline Age (years) & 20 & 15 & 15 & 10 & 10 & 10 & 10 & 10 & 8 & 7 & 7 & 6 & $\mathbf{0}$ \\
\hline $65 \mathrm{kV} 40 \mathrm{mAs}$ & 6.79 & 2.74 & 12.89 & 5.16 & 5.35 & 4.62 & 5.82 & 0.73 & 5.02 & 4.64 & 6.23 & 6.44 & 2.26 \\
\hline $70 \mathrm{kV} 25 \mathrm{mAs}$ & 6.73 & 2.74 & 12.29 & 5.07 & 5.26 & 4.42 & 5.61 & 0.99 & 4.75 & 4.52 & 6.07 & 6.13 & 2.66 \\
\hline $75 \mathrm{kV} 20 \mathrm{mAs}$ & 6.67 & 2.87 & 11.98 & 5.12 & 5.30 & 4.44 & 5.74 & 1.14 & 4.61 & 4.68 & 6.04 & 5.37 & 2.71 \\
\hline $80 \mathrm{kV} 14 \mathrm{mAs}$ & 6.39 & 5.03 & 11.24 & 4.73 & 4.47 & 4.27 & 5.29 & 0.94 & 4.64 & 4.40 & 5.77 & 5.63 & 2.55 \\
\hline $85 \mathrm{kV} 10 \mathrm{mAs}$ & 6.60 & 5.45 & 11.01 & 4.91 & 4.06 & 4.19 & 5.37 & 1.28 & 4.71 & 4.68 & 5.91 & 5.86 & 2.98 \\
\hline 90kV 8mAs & 6.50 & 4.89 & 10.60 & 4.74 & 4.48 & 3.85 & 5.31 & 1.34 & 4.42 & 1.70 & 5.64 & 5.73 & 2.87 \\
\hline $95 \mathrm{kV} 6.3 \mathrm{mAs}$ & 6.38 & 4.48 & 9.91 & 4.49 & 4.85 & 3.67 & 5.03 & 1.26 & 4.32 & 4.30 & 5.56 & 5.26 & 2.81 \\
\hline $100 \mathrm{kV} 5.6 \mathrm{mAs}$ & 6.76 & 4.47 & 10.12 & 4.66 & 5.01 & 4.19 & 5.29 & 1.48 & 4.54 & 4.63 & 5.57 & 5.69 & 3.07 \\
\hline $105 \mathrm{kV} 4.5 \mathrm{mAs}$ & 7.46 & 3.97 & 13.12 & 4.74 & 5.31 & 4.32 & 5.84 & 2.07 & 4.71 & 4.70 & 5.77 & 5.65 & 3.40 \\
\hline $110 \mathrm{kV} 4 \mathrm{mAs}$ & 7.05 & 3.31 & 12.37 & 3.88 & 4.95 & 3.34 & 5.22 & 1.48 & 4.05 & 4.24 & 5.37 & 5.50 & 2.63 \\
\hline
\end{tabular}

Table 3 Percentage decrease in IAK following attenuation by the mattress 


\section{Percentage decrease in IAK from base line (no mattress)}

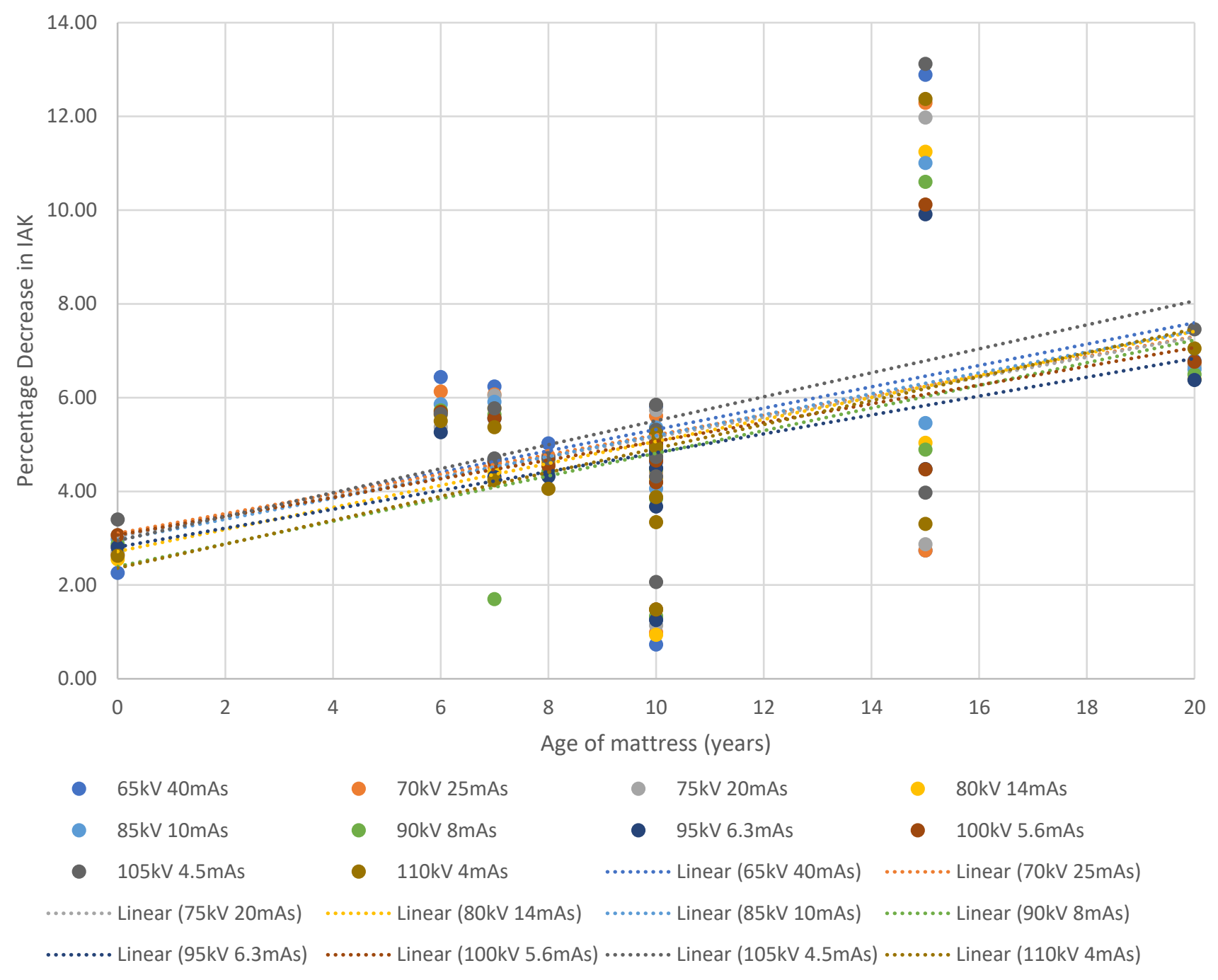

Figure 4 Graph illustrating the trend of increasing attenuation with the age of the mattress

\begin{tabular}{|c|c|}
\hline Exposure factors & Correlation \\
\hline $65 \mathrm{kV} 40 \mathrm{mAs}$ & 0.39 \\
$70 \mathrm{kV} 25 \mathrm{mAs}$ & 0.38 \\
$75 \mathrm{kV} 20 \mathrm{mAs}$ & 0.41 \\
$80 \mathrm{kV} 14 \mathrm{mAs}$ & 0.49 \\
$85 \mathrm{kV} 10 \mathrm{mAs}$ & 0.49 \\
$90 \mathrm{kV} 8 \mathrm{mAs}$ & 0.51 \\
$95 \mathrm{kV} 6.3 \mathrm{mAs}$ & 0.49 \\
$100 \mathrm{kV} 5.6 \mathrm{mAs}$ & 0.49 \\
$105 \mathrm{kV} 4.5 \mathrm{mAs}$ & 0.47 \\
$110 \mathrm{kV} 4 \mathrm{mAs}$ & 0.47 \\
\hline
\end{tabular}

Table 4 Correlation of decrease in IAK and the age of the mattress 
The effect on image quality, measured as IQFinv is shown in Table 5 . The percentage decrease from the base line is shown in Table 6 . There is no/small correlation between mattress age and the decrease in image quality (Table 7).

\begin{tabular}{|c|c|r|r|r|r|r|r|r|r|r|r|r|r|r|}
\hline & \multicolumn{10}{|c|}{ IQF inv } \\
\cline { 2 - 11 } Age (years) & Without & $\mathbf{2 0}$ & $\mathbf{1 5}$ & $\mathbf{1 5}$ & $\mathbf{1 0}$ & $\mathbf{1 0}$ & $\mathbf{1 0}$ & $\mathbf{1 0}$ & $\mathbf{1 0}$ & $\mathbf{8}$ & $\mathbf{7}$ & $\mathbf{7}$ & $\mathbf{6}$ & $\mathbf{0}$ \\
\hline 65kV 40mAs & 2.45 & 2.36 & 1.97 & 2.05 & 2.37 & 2.34 & 2.39 & 2.41 & 2.39 & 2.33 & 2.19 & 2.43 & 2.06 & 2.28 \\
70kV 25mAs & 2.27 & 2.22 & 1.63 & 1.65 & 2.24 & 1.80 & 2.16 & 2.23 & 2.27 & 2.16 & 1.74 & 2.12 & 1.83 & 1.92 \\
75kV 20mAs & 2.24 & 2.15 & 1.74 & 1.77 & 2.20 & 1.91 & 2.21 & 2.12 & 2.14 & 2.23 & 2.00 & 2.10 & 1.72 & 1.94 \\
80kV 14mAs & 2.02 & 2.13 & 1.60 & 1.80 & 2.01 & 1.72 & 2.01 & 2.00 & 2.01 & 1.93 & 1.65 & 1.96 & 1.68 & 1.85 \\
85kV 10mAs & 1.92 & 1.85 & 1.71 & 1.57 & 1.81 & 1.51 & 1.90 & 1.89 & 1.61 & 1.86 & 1.57 & 1.80 & 1.34 & 1.47 \\
90kV 8mAs & 1.83 & 1.68 & 1.40 & 1.48 & 1.63 & 1.59 & 1.73 & 1.78 & 1.67 & 1.72 & 1.52 & 1.75 & 1.35 & 1.54 \\
95kV 6.3mAs & 1.74 & 1.68 & 1.06 & 1.29 & 1.49 & 1.46 & 1.57 & 1.66 & 1.74 & 1.66 & 1.47 & 1.74 & 1.22 & 1.52 \\
100kV 5.6mAs & 1.73 & 1.60 & 1.10 & 1.41 & 1.59 & 1.41 & 1.69 & 1.70 & 1.59 & 1.61 & 1.46 & 1.64 & 1.50 & 1.46 \\
105kV 4.5mAs & 1.73 & 1.58 & 1.23 & 1.24 & 1.50 & 1.39 & 1.61 & 1.64 & 1.55 & 1.51 & 1.36 & 1.62 & 1.11 & 1.50 \\
110kV 4mAs & 1.72 & 1.45 & 1.30 & 1.26 & 1.57 & 1.46 & 1.53 & 1.66 & 1.60 & 1.58 & 1.50 & 1.54 & 1.08 & 1.40 \\
\hline
\end{tabular}

Table 5 Calculated IQF $F_{\text {inv }}$ without and with mattress present across the range of exposure factors

\begin{tabular}{|c|r|r|r|r|r|r|r|r|r|r|r|r|r|r|}
\hline \multicolumn{10}{|c|}{ Percentage decrease in IQFinv due to the mattress } \\
\hline Age (years) & $\mathbf{2 0}$ & $\mathbf{1 5}$ & $\mathbf{1 5}$ & $\mathbf{1 0}$ & $\mathbf{1 0}$ & $\mathbf{1 0}$ & $\mathbf{1 0}$ & $\mathbf{1 0}$ & $\mathbf{8}$ & $\mathbf{7}$ & $\mathbf{7}$ & $\mathbf{6}$ & $\mathbf{0}$ \\
\hline 65kV 40mAs & 3.7 & 19.6 & 16.3 & 3.3 & 4.5 & 2.4 & 1.6 & 2.4 & 4.9 & 10.6 & 0.8 & 15.9 & 6.9 \\
70kV 25mAs & 2.2 & 28.2 & 27.3 & 1.3 & 20.7 & 4.8 & 1.8 & 0.0 & 4.8 & 23.3 & 6.6 & 19.4 & 15.4 \\
75kV 20mAs & 4.0 & 22.3 & 21.0 & 1.8 & 14.7 & 1.3 & 5.4 & 4.5 & 0.4 & 10.7 & 6.3 & 23.2 & 13.4 \\
80kV 14mAs & -0.5 & 20.8 & 10.9 & 0.5 & 14.9 & 0.5 & 1.0 & 0.5 & 4.5 & 18.3 & 3.0 & 16.8 & 8.4 \\
85kV 10mAs & 3.6 & 10.9 & 18.2 & 5.7 & 21.4 & 1.0 & 1.6 & 16.1 & 3.1 & 18.2 & 6.2 & 30.2 & 23.4 \\
90kV 8mAs & 8.2 & 23.5 & 19.1 & 10.9 & 13.1 & 5.5 & 2.7 & 8.7 & 6.0 & 16.9 & 4.4 & 26.2 & 15.8 \\
95kV 6.3mAs & 3.4 & 39.1 & 25.9 & 14.4 & 16.1 & 9.8 & 4.6 & 0.0 & 4.6 & 15.5 & 0.0 & 29.9 & 12.6 \\
100kV 5.6mAs & 7.5 & 36.4 & 18.5 & 8.1 & 18.5 & 2.3 & 1.7 & 8.1 & 6.9 & 15.6 & 5.2 & 13.3 & 15.6 \\
105kV 4.5mAs & 8.7 & 28.9 & 28.3 & 13.3 & 19.7 & 6.9 & 5.2 & 10.4 & 12.7 & 21.4 & 6.4 & 35.8 & 13.3 \\
110kV 4mAs & 15.7 & 24.4 & 26.7 & 8.7 & 15.1 & 11.0 & 3.5 & 7.0 & 8.1 & 12.8 & 10.5 & 37.2 & 18.6 \\
\hline
\end{tabular}




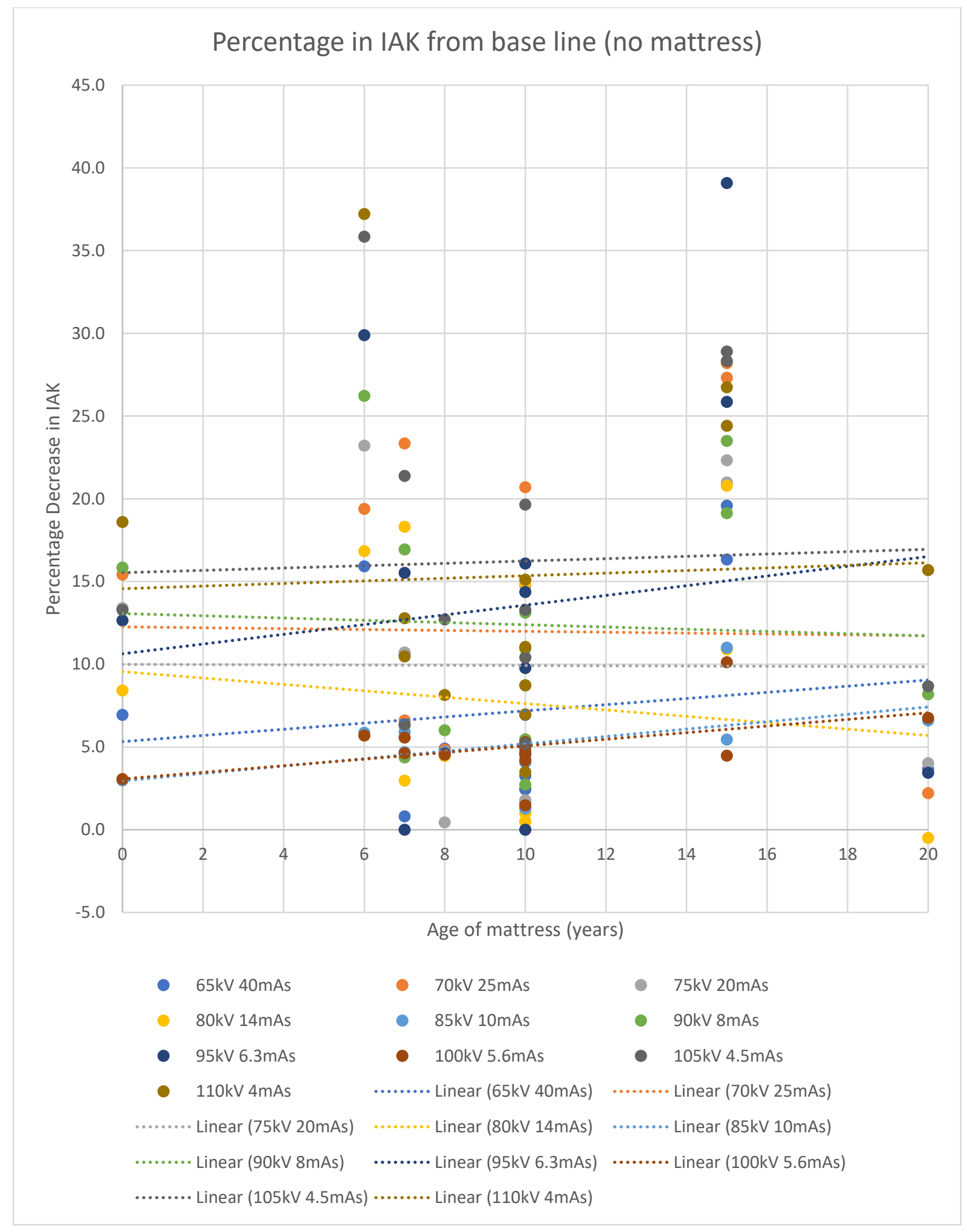




\begin{tabular}{|l|r|}
\hline Age (years) & Correlation \\
\hline $65 \mathrm{kV} 40 \mathrm{mAs}$ & 0.14 \\
$70 \mathrm{kV} 25 \mathrm{mAs}$ & -0.01 \\
$75 \mathrm{kV} 20 \mathrm{mAs}$ & 0.00 \\
$80 \mathrm{kV} 14 \mathrm{mAs}$ & -0.12 \\
$85 \mathrm{kV} 10 \mathrm{mAs}$ & -0.41 \\
$90 \mathrm{kV} 8 \mathrm{mAs}$ & -0.04 \\
$95 \mathrm{kV} 6.3 \mathrm{mAs}$ & 0.12 \\
$100 \mathrm{kV} 5.6 \mathrm{mAs}$ & 0.16 \\
$105 \mathrm{kV} 4.5 \mathrm{mAs}$ & 0.04 \\
$110 \mathrm{kV} 4 \mathrm{mAs}$ & 0.04 \\
\hline
\end{tabular}

Table 7 Correlation between decrease in IQFinv and mattress age

\section{Discussion}

Radiation attenuation and impact on image quality are two aspects of mattress performance that have been evaluated in this study. This research sits alongside the evaluation of the ability of the mattresses to redistribute pressure of pressure ulcer jeopardy areas that has been published previously (10). This research has shown that the addition of a mattress results in the absorption of the primary beam $(0.73 \%$ to $13.12 \%$ reduction in IAK) and a deterioration in image quality as measured by the IQFinv of $-0.50 \%$ to $39.08 \%$ across a range of exposure factors. (The $-0.5 \%$ indicates a slight improvement in image quality but this is within error [8]).

Analysis shows that as the age of the mattress increases the attenuation of the $\mathrm{X}$-ray beam increases across all exposure parameters used in this study. As noted, there was a good/moderate correlation between the decrease in IAK and the age of the mattress (Table 4). To counter this absorption, the operator could increase their exposure factors to ensure the image receptor would receive the same dose. The linear relationship between dose and tube current (mAs) allows the calculation of an $\mathrm{mAs}$ that would be required to ensure comparable image receptor dose. It was found that clinically these differences are insignificant as the change in mAs to compensate for the attenuation would be between 0.01 and $0.13 \mathrm{mAsPractically,} \mathrm{it} \mathrm{is} \mathrm{unlikely} \mathrm{any} \mathrm{X}$-ray equipment would have this level of precision when setting mAs values for bucky work.

The attenuation element of the experiment was carried out without any attenuating material present. This material would cause beam hardening and therefore would alter the properties of the $\mathrm{X}$-ray beam. It is anticipated that this beam hardening would have minimal impact on the findings due to the demonstrated nominal attenuation of the of the mattresses. Further research by repeating the experiment with the presence of the PMMA block used in the image quality component could be performed. Arguably, this would be more representative of clinical practice. IQFinv figures show an overall deterioration in image quality when the mattress was added. There was no correlation between the age of the mattress and the image quality suggesting a quality control programme monitoring changes in image quality over time is not required. The changes in the image quality found in this research would be imperceptible to an observer (6).

This study was not longitudinal and mattresses across a range of ages were used. The age of the mattress was used as an indicator or wear but differences in material used, with older material being rather than or deterioration through repeated use causing a decrease in image quality. Unfortunately, this data was not available so conclusions cannot be made on this without a prospective longer-term study being undertaken. 


\section{Conclusion}

The role of mattresses in radiology is to ensure patient comfort and safety and should be able to do this by minimally attenuating the X-ray photons and not affect image quality. It has been demonstrated that mattresses have a clinically insignificant impact on the primary beam and the image quality measured through IQFinv. While age does correlate with attenuation, it does not with image quality. However, clinically, the age of the mattress has no impact on the exposure factors an operator would select, the mAs delivered by an automatic exposure control system, or the perceptible quality of an image. It is suggested that regular quality assurance of mattress performance may not be required for attenuation properties or impact on image quality. However, this method may have uses in the evaluation of new materials and mattress construction. It would provide manufacturers and potential users specifications on new products.

\section{References}

1. Alresheedi N, Walton LA, Tootell A, Webb JA, Hogg P. A Phantom-Based Method to Assess XRay Table Mattress Interface Pressures. J Med Imaging Radiat Sci [Internet]. 2020 [cited 2020 Jun 22];0(0). Available from: http://www.jmirs.org/article/S1939865420300473/fulltext

2. The lonising Radiations Regulations [Internet]. Queen's Printer of Acts of Parliament; 2017. Available from: http://www.legislation.gov.uk/uksi/2017/1075/contents/made

3. Hiles PA, Institute of Physics and Engineering in Medicine (Great Britain), College of Radiographers (Great Britain), Health Protection Agency (Great Britain). Radiation Protection Division. Recommended standards for the routine performance testing of diagnostic X-ray imaging systems. Report 91. Institute of Physics and Engineering in Medicine; 2005. 112 p.

4. Institute of Physics and Engineering in Medicine (Great Britain). Measurement of the performance characteristics of diagnostic x-ray systems : digital imaging systems. Report 32 Part II. Institute of Physics and Engineering in Medicine; 2010. 125 p.

5. Pascoal A, Lawinski CP, Honey I, Blake P. Evaluation of a software package for automated quality assessment of contrast detail images - comparison with subjective visual assessment. Phys Med Biol [Internet]. 2005 Dec 7;50(23):5743-57. Available from: http://stacks.iop.org/00319155/50/i=23/a=023?key=crossref.6cd2a5ac86c27396672e174f6f8d2d90

6. Geso M, Shanahan M, Alghamdi SS, Davidson R, Alghamdi S. Low-Contrast Detail Phantom: Proof of Concept. J Med Imaging Radiat Sci. 2016 Mar 1;47(1):60-5.

7. BV R van der B-TNAMS, 2006 undefined. Manual CDRAD Analyser.

8. Konst B, Weedon-Fekjær H, Båth M. Image quality and radiation dose in planar imaging Image quality figure of merits from the CDRAD phantom. J Appl Clin Med Phys [Internet]. 2019 Jul 1 [cited 2020 Oct 14];20(7):151-9. Available from: /pmc/articles/PMC6612684/?report=abstract

9. Konst B, Weedon-Fekjær H, Båth M. Image quality and radiation dose in planar imaging Image quality figure of merits from the CDRAD phantom. J Appl Clin Med Phys. 2019 Jul;20(7):151-9.

10. Alresheedi N, Walton LA, Tootell A, Webb JA, Hogg P. A Phantom-Based Method to Assess XRay Table Mattress Interface Pressures. J Med Imaging Radiat Sci. 2020;0(0). 